\title{
Correction to: Comparing the impacts of an invasive grass on nitrogen cycling and ammonia-oxidizing prokaryotes in high-nitrogen forests, open fields, and wetlands
}

Tyler M. Rippel (D) Christine L. Iosue • Pasquale J. Succi • Dennis D. Wykoff • Samantha K. Chapman

Published online: 25 March 2020

(C) Springer Nature Switzerland AG 2020

\section{Correction to: Plant and Soil}

https://doi.org/10.1007/s11104-02004458-8

There is a formatting error which impacts the interpretation of Table 2. Some equations in the "Predictor Variables and Standardized Beta Estimates" section in Table 2 are not correctly associated with their correct "Model Rank" due to these formatting issues. A newly formatted Table is below.

Publisher's note Springer Nature remains neutral with regard to jurisdictional claims in published maps and institutional affiliations.

The online version of the original article can be found at https://doi.org/10.1007/s11104-020-04458-8

C. L. Iosue · P. J. Succi - D. D. Wykoff · S. K. Chapman Department of Biology, Villanova University, 800 Lancaster Ave., Villanova, PA 19085, USA

Present Address:

T. M. Rippel ( $\bowtie)$

Georgetown University, 3700 O St NW, Washington, DC 20057, USA

e-mail: tr599@georgetown.edu 
Table 2 Best-fit models from linear mixed model selection with the relative abundance of AOA amoA and the relative abundance of AOB amoA as response variables.

\begin{tabular}{|c|c|c|c|c|c|}
\hline $\begin{array}{l}\text { Response } \\
\text { Variable }\end{array}$ & $\begin{array}{l}\text { Model } \\
\text { Rank }\end{array}$ & AIC & $\begin{array}{l}\mathrm{P} \\
\text { Value }\end{array}$ & $\begin{array}{l}\text { Intercept } \\
( \pm \mathrm{se})\end{array}$ & Predictor Variables and Standardized $\beta$ estimates $( \pm$ se $)$ \\
\hline \multirow[t]{4}{*}{$\begin{array}{c}\text { Archaeal } \\
\text { amoA }\end{array}$} & 1 & 94.0 & 0.001 & $9.4 \pm 1.3$ & $\begin{array}{l}\mathbf{a N H}_{4}^{+}(-0.24 \pm 0.16)+\mathbf{S M}(-0.28 \pm 0.18)+\mathbf{B D}^{\bullet}(-0.32 \pm 0.16)+\mathbf{p H}^{*}(-0.37 \pm 0.15)+ \\
\quad \mathbf{M V B}^{\bullet}(-0.33 \pm 0.16)\end{array}$ \\
\hline & 2 & 94.2 & 0.012 & $8.7 \pm 1.4$ & $\mathbf{S M}(-0.28 \pm 0.19)+\mathbf{B D}(-0.32 \pm 0.17)+\mathbf{p H}(-0.37 \pm 0.17)+\mathbf{M V B}(-0.23 \pm 0.17)$ \\
\hline & 3 & 94.2 & 0.012 & $8.5 \pm 1.3$ & $\mathbf{a N H}_{\mathbf{4}}{ }^{+}(-0.22 \pm 0.14)+\mathbf{p H}(-0.36 \pm 0.16)+\mathbf{B D}(-0.19 \pm 0.14)+\mathbf{M V B}(-0.24 \pm 0.17)$ \\
\hline & 4 & 95.1 & 0.012 & $9.1 \pm 1.5$ & $\mathbf{a N H}_{4}{ }^{+}(-0.12 \pm 0.14)+\mathbf{S M}(-0.18 \pm 0.19)+$ Density $(-0.29 \pm 0.17)+\mathbf{p H}(-0.48 \pm 0.18)$ \\
\hline \multirow{4}{*}{$\begin{array}{r}\text { Bacterial } \\
\text { amoA }\end{array}$} & 1 & 142.7 & 0.012 & $6.0 \pm 2.0$ & $\mathbf{p H}^{\bullet}(-0.29 \pm 0.15)+\mathbf{p N H}_{\mathbf{4}}^{+\bullet}(0.25 \pm 0.14)$ \\
\hline & 2 & 143.4 & 0.018 & $5.8 \pm 1.9$ & BD $(-0.17 \pm 0.15)+\mathbf{p H}(-0.21 \pm 0.16)+\mathbf{p N H}_{\mathbf{4}}^{+}(0.30 \pm 0.15)$ \\
\hline & 3 & 143.4 & 0.017 & $7.3 \pm 2.2$ & $\mathbf{B D}(-0.25 \pm 0.16)+\mathbf{p H}(-0.26 \pm 0.16)+\mathbf{R o o t s}(-0.21 \pm 0.15)+\mathbf{p N H}_{\mathbf{4}}{ }^{+}(0.30 \pm 0.15)$ \\
\hline & 4 & 143.6 & 0.015 & $7.4 \pm 1.9$ & $\mathbf{p H}(-0.37 \pm 0.14)$ \\
\hline
\end{tabular}

Symbols: $\mathbf{a N H}_{\mathbf{4}}{ }^{+}=$resin-available $\mathrm{NH}_{4}{ }^{+}, \mathbf{p N H}{ }_{\mathbf{4}}{ }^{+}=$soil $\mathrm{NH}_{4}{ }^{+}$pool, $\mathbf{B D}=$ bulk density, $\mathbf{p H}=$ soil $\mathrm{pH}, \mathbf{M V B}=$ M. vimineum biomass, $\mathbf{S M}=$ soil moisture content. The full list of predictor variables input for each response variable is located in the Materials and Methods. Significance: $* * *=p<0.001, * *=p<0.01, *=\mathrm{p}<0.05,{ }^{\bullet}=\mathrm{p}<0.1$

The original article has been corrected. 